\title{
Gonzalo's books : La république des Cannibales, de Montaigne à Shakespeare
}

\section{Frank Lestringant}

\section{(2) OpenEdition \\ Journals}

Édition électronique

URL : http://journals.openedition.org/shakespeare/170

DOI : 10.4000/shakespeare. 170

ISSN : 2271-6424

Éditeur

Société Française Shakespeare

Édition imprimée

Date de publication : 1 novembre 2004

Pagination : 175-193

ISBN : 2-9521475-0-7

\section{Référence électronique}

Frank Lestringant, «Gonzalo's books : La république des Cannibales, de Montaigne à Shakespeare », Actes des congrès de la Société française Shakespeare [En ligne], 21 | 2004, mis en ligne le 01 février 2007, consulté le 30 avril 2019. URL : http://journals.openedition.org/shakespeare/170 ; DOI :

10.4000/shakespeare. 170 


\section{Shakespeare et Montaigne vers un nouvel humanisme}

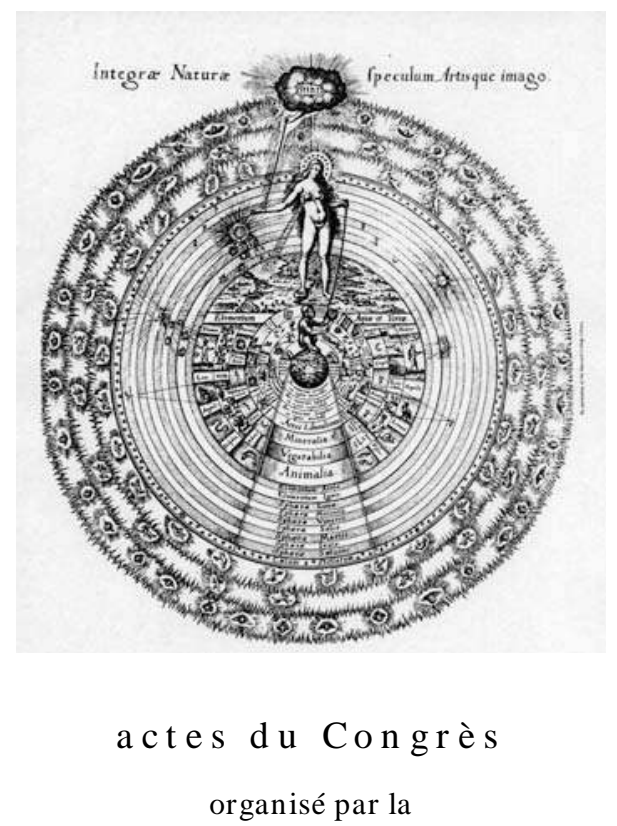

SOCIÉTÉ FR ANÇAISE SH AKESPEARE en collaboration avec la

S OC I É T É I N T E R N A T I O N A L E DES AM I D E M O N A I G N les 13,14 et 15 mars 2003

$$
\begin{gathered}
\text { textes réunis par } \\
\text { Pierre KAPITANIAK } \\
\text { sous la direction de } \\
\text { Jean-Marie MAGUIN }
\end{gathered}
$$




\section{COMITÉ SCIENTIFIQUE :}

Margaret Jones-Davis

Gisèle Venet

Jean-Marie Maguin

Yves Peyré

François Laroque

Pierre Kapitaniak

\section{COUVERTURE :}

Robert Fludd

Utriusque Cosmi Historia (1617-19)

planche 17

conception graphique et logo

Pierre Kapitaniak

\section{(C) 2003 Société Française Shakespeare}

Institut du Monde Anglophone

Université de Paris III - Sorbonne Nouvelle

http:// univ-montp3.fr/SFS/

5 rue de l'École de Médecine

75006 Paris

Diffusion :

AVL DIFFUSION

Parc Euromédecine

34198 MONTPELLIER CEDEX 5

ISBN 2-9521475-0-7

Tous droits de traduction, de reproduction et d'adaptation réservés pour tous les pays 


\title{
GONZALO'S BOOKS : \\ LA RÉPU B LIQUE DES CANNIBALES, De Montaigne À SHAKespeare
}

\author{
Frank LESTRINGANT
}

\begin{abstract}
Dans La Tempête, on le sait, Shakespeare s'inspire directement d'un passage de Montaigne. Une tirade de Gonzalo, à l'acte II, scène i, provient en droite ligne du chapitre "Des Cannibales ", que Shakespeare a lu dans la traduction de John Florio de 1603. En revenant sur cet emprunt presque littéral, depuis longtemps établi et qui ne prête pas à discussion, mon propos sera double: je souhaiterais d'une part éclairer rétroactivement le chapitre "Des Cannibales" par l'usage dramatique qu'en propose Shakespeare; en second lieu, il s'agira de réévaluer le rôle de Gonzalo à partir de Montaigne ; autrement dit, de réhabiliter Gonzalo et sa culture livresque.

Gonzalo's Books: The Cannibals' Republic from Montaigne to Shakespeare In The Tempest Shakespeare is directly inspired by a passage from Montaigne. One of Gonzalo's speeches, act II, scene i, comes straight from the chapter on "The Cannibals", that Shakespeare had read in Florio's translation of 1603. By revisiting this almost literal borrowing, long established and unquestioned, my purpose is double: first, I would like to propose a new reading of "The Cannibals" in the retroactive light of its dramatic use offered by Shakespeare; secondly, I will endeavour to reassess Gonzalo's role from Montaigne's reading - in other words, to rehabilitate Gonzalo and his scholarly culture.
\end{abstract}

\section{Formule négative}

$\mathbf{J}$ e partirai de la formule négative 249 et de l'emprunt de Shakespeare à Montaigne, par la voix du vieux lord Gonzalo, un idéaliste un peu gâteux, au demeurant conseiller fort honnête du roi de Naples Alonso. Voici ce passage célèbre du chapitre «Des Cannibales »:

C'est une nation, dirai-je à Platon, en laquelle il n'y a aucune espèce de trafic; nulle connaissance de lettres; nulle science de nombres; nul nom de magistrat, ni de supériorité politique ; nul usage de service, de richesse, ou de pauvreté; nuls contrats; nulles successions; nuls partages ; nulles occupations, qu'oisives; nul respect de parenté, que commun; nuls vêtements; nulle agriculture; nul métal; nul usage de vin ou de blé. Les paroles mêmes, qui signifient le mensonge, la trahison, la dissimulation, l'avarice, l'envie, la détraction ${ }^{250}$, le pardon,

${ }^{249}$ Sur la «formule négative », cette figure privilégiée et obligée du discours primitiviste, voir Harry Levin, The Myth of the Golden Age in the Renaissance, Londres, Faber \& Faber, 1970, p. 11; Gérard Defaux, Marot, Rabelais, Montaigne: l'écriture comme présence, Paris, Champion, 1987, p. 172 ; Christian Marouby, Utopie et primitivisme. Essai sur l'imaginaire anthropologique à l'âge classique, Paris, Éditions du Seuil, 1990, p. 113-126: «Rhétorique de la négativité ».

${ }^{250}$ La calomnie. 
inouïes. Combien trouverait-il la république qu'il a imaginée, éloignée de cette perfection : viri a diis recentes ${ }^{251}$ ?

Dans sa version anglaise de 1603 , John Florio traduit au plus près du texte :

It is a nation, would I answere Plato, that hath no kinde of traffike, no knowledge of Letters, no intelligence of numbers, no name of magistrate, nor of politike superioritie; no use of service, of riches, or of poverty; no contracts, no successions, no dividences, no occupation but idle; no respect of kinred, but common, no apparrell but naturall, no manuring of lands, no use of wine, corne, or mettle. The very words that import lying, falshood, treason, dissimulation, covetousnes, envie, detraction, and pardon, were never heard-of amongst them. How dissonant would hee finde his imaginary common-wealth from this perfection $^{252}$ ?

Et voici ce que Shakespeare fait de ce passage dans La Tempête:

Gonzalo. I'th' commonwealth I would by contraries

Execute all things; for no kind of traffic

Would I admit, no name of magistrate ;

Letters should not be known ; riches, poverty

And use of service, none ; contract, succession,

Bourn, bound of land, tilth, vineyard, none ;

No use of metal, corn, or wine, or oil ;

No occupation : all men idle, all ;

And women too - but innocent and pure ;

No sovereignty -

[...]

All things in common nature should produce

Without sweat or endeavour: treason, felony,

Sword, pike, knife, gun, or need of any engine,

Would I not have; but nature should bring forth

Of it own kind, all foison, all abundance,

To feed my innocent people.

[...]

I would with such perfection govern, sir,

T'excel the Golden $\mathrm{Age}^{253}$.

Shakespeare, on le constate, glose à peine la traduction de Florio, mais il anime le texte d'origine en le dissociant en plusieurs répliques.

251 Sénèque, Lettres à Lucilius, XC : «Hommes fraîchement sortis de la main des dieux ».

252 Montaigne, The Essayes, or Morall, Politike and Millitarie Discourses of Lo: Michaell de Montaigne [...]. First written by him in French. And now done into English, Londres, 1603, "The thirtieth Chapter. Of the Caniballes », p. 102. J'ai eu recours au fac-similé publié par The Scolar Press Limited (Menston, England) en 1969.

253 Shakespeare, La Tempête / The Tempest, II.i.147 sqq. Je renvoie à l'édition et traduction de Joseph Aynard, Paris, Les Belles Lettres, 1927 et 1967. 
L'attaque de la première tirade place l'ensemble du tableau sous le signe du monde renversé, tandis que la dernière réplique convoque le modèle mythologique de l'âge d'or. Cette double référence, l'une populaire et l'autre savante, jette la suspicion sur ce propos emprunté. La société idéale de Gonzalo oscille entre le carnaval et le songe humaniste.

La situation dramatique de ce début de l'acte II et le rapport entre les personnages accentuent le caractère parodique de cette reprise. Gonzalo déclame et rêve tout éveillé. Le fait est qu'il est bien seul dans son rêve. Face à lui, les autres personnages de la scène s'amusent ou s'impatientent. Sébastien et Antonio rient sous cape, puis de plus en plus ouvertement. Inquiet quant au sort de son fils Ferdinand disparu pendant la tempête, le roi Alonso ronge son frein.

Dans la paraphrase de Montaigne par Shakespeare, le plus simple serait donc de conclure à un démenti cinglant. La reprise ironique de la formule négative, dont se gaussent les autres personnages, à l'exception d'Alonso qui s'en irrite, apparaîtrait alors comme une gifle adressée à l'idéaliste Montaigne. Le ridicule qui tombe sur Gonzalo, doux rêveur radotant un peu, retomberait par ricochet sur l'auteur des Essais. Shakespeare serait alors un lecteur pressé et désinvolte, découpant, au mépris du contexte, une période pour en faire une tirade, et de surcroît la lacérant en plusieurs morceaux entrecoupés de lazzi, faisant des gorges chaudes d'un extrait mutilé, privé de ses résonances et de son ambiguïté. C'est la lecture la plus simple de cet emprunt de Shakespeare à Montaigne, mais pas forcément la bonne.

Il en existe en effet une autre, qui exige le retour à Montaigne comme préalable à la lecture de Shakespeare, et c'est celle que je vais tenter d'exposer.

Que Montaigne a-t-il bien voulu dire ? Ou plutôt que dit-il en fait quand il reprend à son compte la «formule négative»? Cette formule négative est elle-même profondément ambiguë. On la rencontre dans les contextes les plus opposés, chez les adversaires les plus inconciliables, ceux qui, comme Montaigne apparemment, célèbrent dans la société indienne l'heureuse survivance de l'âge d'or ; ceux qui, au contraire, invitent à mépriser ces hommes incapables de maîtriser les arts et techniques qui rendent cette vie sur terre tout juste supportable. Aux adeptes du primitivisme doux s'opposent ceux du 
primitivisme dur ; aux admirateurs inconditionnels des peuples nus les colonisateurs les plus agressifs et les plus méprisants. Or les uns et les autres ont en partage la même rhétorique du «ni...ni...». Ils ont en partage le même slogan, cette litanie privative qui se retrouve de Gomara $^{254}$ à Montaigne et de Cortès à Ronsard. La construction rhétorique implique une ambivalence profonde, dont, de toute évidence, Shakespeare a su jouer magistralement.

Mais Montaigne n'en joue-t-il pas lui-même dans le chapitre I, 31 des Essais? Seul un regard distrait pourrait voir en Montaigne un Gonzalo avant la lettre, du moins le Gonzalo tout juste ébauché du début de la pièce, et qui va prendre ensuite consistance et épaisseur. Ce premier Gonzalo n'est pas loin du personnage comique du pedante dont le savoir indiscrètement étalé fait rire à ses dépens. En témoigne le «widow Dido», repris en échos parodiques par Antonio et Sébastien, refrain comique, paronomase grotesque, sorte de jingle loufoque dont la sonorité intrigue avant de faire rire aux éclats ${ }^{255}$. Gonzalo, il est vrai, va progressivement se métamorphoser en un sage, «this Sir Prudence» sur lequel ironisent les diaboliques Antonio et Sébastien ${ }^{256}$, le double de Prospero en quelque sorte, en plus humain et en plus compassionnel. À l'instar de Prospero, Gonzalo est capable de percer à jour les plus noirs secrets. Mais sa lucidité extrême sur le fond de la nature humaine, qui révèle en lui un pessimiste inattendu, n'empêche qu'il fait figure d'abord, dans la pièce, de doux rêveur.

\section{Degrés}

Rien donc n’indique que Montaigne adhère tout uniment au cliché de la formule négative. Tout indique en fait le contraire.

Le texte des Essais est beaucoup plus rusé qu'il n'en a l'air, et Shakespeare, comme on va le voir, a parfaitement saisi cette ruse, qu'il prolonge et reconduit à sa manière. La formule négative, dans ce passage précis du chapitre «Des Cannibales », est introduite par une

${ }^{254}$ Francisco Lopez de Gomara, Histoire générale des Indes Occidentales, II, 96, trad. Martin Fumée, Paris, 1587, $\mathrm{f}^{\circ} 188 \mathrm{v}^{\circ}-189 \mathrm{r}^{\circ}$ : « Des choses nécessaires, desquelles avaient faute ces Mexicains ».

255 Shakespeare, La Tempête/ The Tempest, II.i.68-91.

256 Ibid., II.i.270. 
supposition, et cette supposition est capitale, car elle relativise tout ce qui va suivre : «C'est une nation, diroy je à Platon, en laquelle...» 257.

Diroy je. Le futur a valeur ici de conditionnel. Il est justement rendu dans une récente édition modernisée par Dirais-je ${ }^{258}$. Montaigne dit qu'il dit, ou plutôt qu'il dira ou qu'il dirait, mais il ne dit pas nûment, ce qui obligerait à mettre à son compte et à lui attribuer sans restriction la série de négations qui suit immédiatement. On sait que Montaigne joue constamment et sciemment des degrés du langage. C'est de ce point de vue un expert en bathmologie, cette «science des échelonnements du langage» que Roland Barthes appelait de ses vœu ${ }^{259}$. Qu'il s'agisse des Essais ou du Journal de voyage en Italie, la science des degrés que possède Montaigne lui permet d'introduire dans son texte un dialogisme entre des voix situées à différents étages. La bathmologie sert de support à la polyphonie; elle favorise les réverbérations infinies de l'ironie. Grâce à la bathmologie, s’insinue dans la trame serrée des Essais la discontinuité du jeu théâtral. Il est évident que Shakespeare a parfaitement retenu cette suggestion théâtrale contenu dans le «diroy je » montaignien.

Du reste et en l'espèce, l'hypothèse est assez peu vraisemblable. Montaigne a de bien faibles chances de rencontrer un jour Platon et de dialoguer tranquillement avec lui. L'humaniste Montaigne se plaît à imaginer cet échange fictif, qui abolirait l'immense intervalle de vingt siècles de décadence et d'oubli, et comme par magie mettrait un terme à la déperdition d'intelligence et de savoir qui en a résulté. Au fond c'est la même nostalgie qui saisit Gonzalo quand il imagine sa république idéale. Ce rêve qui consisterait à pouvoir converser de plain-pied avec les Anciens, en une joute à bâtons rompus, entre égaux, entre amis, après une aussi longue absence, c'est le grand rêve de la Renaissance, un rêve définitivement perdu à l'époque de Montaigne et de Shakespeare.

Mais comme Montaigne sait que ce rêve n'est après tout qu'un rêve, il l'accompagne d'une nuance de légère ironie. L'hypothèse introduite par le «diroy je» est incongrue, en effet. Assurément

257 Montaigne, Essais, I, 31, éd. Pierre Villey, Paris, PUF, 1965, p. 206

258 Montaigne, Les Essais, éd. Jean Céard et alii, LGF, Le Livre de Poche, «La Pochothèque », 2001, p. 320

259 Roland Barthes, Roland Barthes par Roland Barthes. Cité par Pierre Laurens, «Monsieur de Montaigne disait que ... (bathmologies)», in F. Lestringant éd., Rhétorique de Montaigne, Paris, Champion, 1985, p. 73-84. 
Platon, transporté au pays des Cannibales, y perdrait son grec. Il serait surpris, à tout le moins, qu'une société, contrairement à celle qu'il a imaginée, puisse «se maintenir avec si peu d'artifice et de soudure humaine» (I, 31, 206). Cinglant démenti apporté, au nom de l'expérience, à la savante et complexe architecture de la République.

\section{Déclamation}

Ce jeu très subtil qui marie l'incon gruité à l'hypothèse d'école renvoie à une tradition rhétorique bien établie, qui est celle de la déclamation. Terme à la fois plus large et plus technique que celui de «paradoxe ${ }^{260}$, la «déclamation» désigne l'exercice de développement oratoire sur un thème donné que les rhéteurs recommandaient pour la formation ou l'entraînement de l'orateur. « Le 'réel irréel', tel est l'objet psychologique, judiciaire et rhétorique » du déclamateur ${ }^{261}$. Dans la déclamation, «les deux notions essentielles, qui sont liées, sont celles d'exercice et de fiction $»^{262}$. La déclamation se définit par sa totale liberté, qui en fait l'instrument privilégié pour une réflexion morale sans préjugé. Le point de vue y est mobile, l'identité du locuteur constamment fuyante.

Pour des raisons qui tiennent à l'instabilité de la période, aux bouleversements qui l'agitent et aux formidables résistances que rencontrent les idées nouvelles, la Renaissance a fait de la déclamation un de ses modes d'expression privilégiés. La déclamation irrigue toute la littérature $\mathrm{du} \mathrm{XVI}^{\mathrm{e}}$ siècle, depuis l'Éloge de la Folie d'Érasme jusqu'aux Essais de Montaigne. Thomas More, dans l'Utopie, a écrit une déclamation à support géographique. L'œuvre de François Rabelais est émaillée de déclamations, éloge des dettes par Panurge, hymne de l'herbe nommée Pantagruélion, louanges de «messere Gaster, premier maître ès arts du monde ». Étienne de La Boétie, l'ami de Montaigne, a composé avec le Discours de la Servitude volontaire

${ }^{260}$ Voir Patrick Dandrey, L'Éloge paradoxal de Gorgias à Molière, Paris, PUF, 1997, p. 137-173: «Montaigne paradoxal». La perspective ici adoptée est tangente à celle que dessine cet ouvrage.

261 Pascal Quignard, Albucius, P.O.L., 1990, et Livre de Poche, 1990, chap. II, p. 15

262 Jacques Chomarat, Grammaire et rhétorique chez Érasme, Paris, Les Belles Lettres, 1981, t. II, p. 935 . 
une déclamation oratoire, fiévreuse et d'une logique implacable ${ }^{263}$. Plusieurs des chapitres ou fragments de chapitres des Essais sont des déclamations ${ }^{264}$. On peut citer l'éloge de la gravelle dans «De l'expérience» (III, 13), ou, cas plus piquant, le plaidoyer en faveur du «membre indocile» dans « De la force de l'imagination» (I, 21). Mais le meilleur exemple de déclamation chez Montaigne est sans conteste «Des Cannibales », apologie des libres anthropophages du Brésil, en qui revivent l'âge d'or des Anciens et la république idéale rêvée par Platon et Plutarque. Le style de la déclamation se signale chez Montaigne dans les hyperboles laudatives et dans des sentences sans réplique comme cette formule où l'on a voulu voir le slogan du relativisme : «Chacun appelle barbarie ce qui n'est pas de son usage». Mais il se reconnaît surtout dans un ton d'ironie tour à tour badine et indignée.

À cet égard, le passage précis que nous étudions relève par excellence de la rhétorique de la déclamation. Il s'inscrit très exactement dans la mouvance de l'Encomium Moriae. Une ligne de folie court d'Érasme à Montaigne, à travers un XVI e siècle qui en a vu bien des formes, souvent plus virulentes et plus tragiques. Cette folie érasmienne est une folie douce, une folie critique qui suppose le dédoublement et le jeu théâtral ${ }^{265}$. Or, entre l'Éloge de la Folie et « Des Cannibales », il existe un relais: La Pazzia (en italien «la Folie»), «traité fort plaisant en forme de Paradoxe ${ }^{266}$. Cet ouvrage anonyme publié à Venise en 1540 a été attribué à Ascanio Persio ou à Vianezio

263 Jean Lafond, «Le Discours de la Servitude volontaire de La Boétie et la rhétorique de la déclamation», Mélanges sur la littérature de la Renaissance à la mémoire de V. L. Saulnier, Genève, Droz, 1984, p. 736. Sur les rapports de la Servitude avec «Des Cannibales », voir mon livre: Le Cannibale, grandeur et décadence, Paris, Perrin, 1994, chap. VIII, p. 181-183.

264 Voir André Tournon, Montaigne. La glose et l'essai, Lyon, Presses Universitaires de Lyon, 1983, p. 203-228

265 Marc Fumaroli, « Microcosme comique et macrocosme solaire : Molière, Louis XIV, et L'Impromptu de Versailles », Revue des Sciences humaines, $\mathrm{t}$. XXXVII, ${ }^{\circ} 145$, janvier-mars 1972, p. 95-114, et notamment p. 95-98.

${ }^{266}$ La Pazzia, Venise, 1540 ; réédition en 1543: «Stampata in Venegia per Giovanni Andrea Vavassore detto Guadagnino et Florio fratello». Traduction française: Les louanges de LA FOLIE, Traicté fort plaisant en forme de Paradoxe, traduict d'Italien en François par feu messire Jean du Thier, Paris, Hertman Barbé, 1566. — Cette source a été identifiée, et proposée avec quelques réserves, par José V. de Pina Martins, "Modèles portugais et italiens de Montaigne", Montaigne et l'Europe. Actes du colloque international de Bordeaux (1992), Mont-de-Marsan, Éditions InterUniversitaires, 1992, p. 139-152. Du même, «Um Livro acerca da Loucoura (La Pazzia, 1540), e a sua discutivel originalidade », Hom enaje a Eugenio Asensio, Madrid, Editorial Gredos, 1988, p. 361-378. 
Albergati ${ }^{267}$. Il introduit, bien avant Montaigne, les peuples nus du Nouveau Monde dans l'espace critique de la déclamation. La Folie parle. Elle peut tout dire, à commencer par prendre l'opinion commune à rebrousse-poil. Elle ne craint pas non plus de démentir les plus savants. Du «peuple nouvellement découvert en l'Indie Occidentale», elle affirme en effet qu'il vivait heureux « sans lois, sans lettres, et sans aucuns sages». Ces bienheureux méprisaient l'or et les «joyaux précieux ». Ils ne connaissaient «ni l'avarice, ni l'ambition, ni quelque autre art que ce fût». Prenant leur nourriture «des fruits que la terre sans artifice produisait», ils «avaient comme en la République de Platon, toutes choses communes, jusques aux femmes et petits enfants: lesquels dès leur naissance ils nourrissaient et élevaient en communauté comme propres » ${ }^{268}$.

L'accord ponctuel avec Platon n'empêche pas un pied de nez à l'égard de celui qui aurait voulu que les philosophes fussent rois, ou, à défaut, que les rois devinssent philosophes. La Pazzia le contredit ouvertement : «Là-dessus je répondrai que non : mais que les peuples ne sauraient être plus malheureux, ni en plus grande calamité, que de se voir tomber ès mains de tels philosophâtres et trop sages hommes ${ }^{269}$. » À preuve les Espagnols qui, «avec leur trop de savoir, leurs grandes finesses, leurs très dures et insupportables lois et édits », ont rempli «de cent mille maux, fâcheries et travaux» cette contrée naguère bénie des dieu $x^{270}$.

La désinvolture avec laquelle le monde exotique est plaqué sur le monde classique se retrouve, on l'a constaté, chez Montaigne. De cette désinvolture, une fois de plus, Platon fait les frais : «C'est une nation, diroy je à Platon, en laquelle...» Ce sens de l'incongruité calculée porte la marque de La Pazzia, Montaigne s'étant contenté d'atténuer l'ironie un peu lourde de son modèle.

\section{Utopie, ou le discours du rien}

L'emprunt ponctuel de Shakespeare à Montaigne nous a donc permis de remonter en amont et par étapes jusqu’à Érasme et More, le

${ }^{267}$ Patrick Dandrey, op. cit., p. 102

268 Les Louanges de la Folie, op. cit., 1566, $\mathrm{f}^{\circ} \mathrm{C} 6 \mathrm{v}^{\circ}$.

${ }^{269}$ Ibid., $\mathrm{f}^{\circ} \mathrm{C} 7 \mathrm{r}^{\circ}$.

270 Ibid. 
morosophe bien nommé, l'auteur de L'Utopie, qui est précisément une déclamation. Par l'intermédiaire de Montaigne, en effet, Shakespeare renoue avec le grand texte d'un autre grand Anglais.

Mais revenons à La Tempête et au rôle de Gonzalo. Alonso, roi de Naples, a marié sa fille Claribel à Tunis en Afrique, et se désole de cet éloignement définitif. De surcroît il a perdu dans la tempête le seul enfant qui lui reste, son fils Ferdinand, qu'il croit noyé. C'est pour le distraire de son deuil et de sa mélancolie, que Gonzalo, sans transition et tout de go, se lance dans l'énoncé de la formule négative. Comme chez Montaigne, c'est une pure supposition, apparemment des plus gratuites et faite par jeu, pour détourner le roi de ses tristes pensées. Or il n'est pas de meilleur moyen pour faire oublier le réel, dans toute sa cruauté, que d'inventer une fiction la plus éloignée possible de toute vraisemblance. C'est précisément l'exercice auquel se livre Gonzalo. Son État idéal n'a pas la moindre chance de se concrétiser un jour :

Had I plantation of this isle, my lord [...]

And were the king on't, what would I do ?

(II.i.138, 141)

Cette peinture est éloignée de tout réalisme. Gonzalo n'est pas roi, il le sait, et il n'a nulle prétention à le devenir. À la différence de ses contradicteurs Antonio et Sébastien, il n'a pas l'intention d'usurper le trône d'Alonso, roi de Naples. Au contraire ce conseiller fidèle va sauver la vie de son prince, en le réveillant à temps, alors qu'il est sur le point d'être frappé par l'épée des deux traîtres. Si Gonzalo est roi, le temps d'un rêve, c'est un roi de papier ou de Carnaval, un roi inoffensif et bienveillant qui abdique toute souveraineté au profit de ses sujets.

Cette peinture invraisemblable, de surcroît, n'offre pas de véritable cohérence. Gonzalo y confond plusieurs modèles difficilement compatibles: celui du monde renversé, évoqué d'entrée de jeu : «I would by contraries / Execute all things »; celui de l'âge d'or, «the golden age », énoncé in fine, et, bien sûr, le rêve de la république idéale. Il semblerait que sur le mode élevé de la déclamation érudite, Gonzalo joue ici le rôle de fou du roi. Un fou noble, a gentle jester, en quelque sorte, par opposition au grotesque Trinculo, qui est un bouffon de bas étage. 
Comme la Folie dans la déclamation d'Érasme et comme Montaigne dans les Essais ${ }^{271}$, Gonzalo se contredit, et ne semble pas s'en apercevoir. Antonio et Sébastien n'ont pas tort de remarquer la contradiction existant entre le début et la fin de sa tirade. Gonzalo voudrait être roi (and were the king on't) d'un peuple où n'existe nulle notion de souveraineté (No sovereignty), de sorte que « The latter end of his commonwealth forgets the beginning» (II.i.147).

C'est d'une manière déjà ce qui se passe dans le chapitre «Des Cannibales », où les contradictions ne sont pas moins évidentes. Les Cannibales, commence par nous dire Montaigne, ignorent toutes les inventions, et partant tous les maux, qui caractérisent notre société. Voici les Cannibales, en l'occurrence les Tupinikin du Rio de Janeiro, dont Thevet, puis Léry avaient divulgué quelques années plus tôt les féroces prouesses, érigés au rang de type idéal et rejoignant «toutes les peintures de quoi la poésie a embelli l'âge doré ». Rien de bien neuf, en vérité, dans cette litanie négative, que Gonzalo dévidera à son tour.

Or Montaigne sait bien que dans la réalité, ces Indiens exercent l'agriculture, filent et tissent le coton, s'adonnent à l'économie de troc, reconnaissent un système de parenté extrêmement complexe et ne cessent de se faire la guerre. Dans la suite du chapitre, du reste, il semble oublier la négation initiale. Quitte à décevoir les rêveurs et les poètes, Montaigne reconstruit alors la figure positive du Brésilien, en convoquant toutes les circonstances matérielles qui le font paraître en corps dans la trame de l'essai : son hamac et ses armes, sa nourriture et son breuvage «fait de quelque racine» et «couleur de nos vins clairets », ses armes, son bâton de rythme, mais aussi sa danse, ses gestes et contenances, sa musique et une poésie amoureuse digne des grâces anacréontiques.

Autrement dit, le lieu commun primitiviste était une simple étape dans le raisonnement, tout comme la référence à la république idéale de Lycurgue et de Platon. La formule négative représente le moment de la table rase, à partir duquel la reconstruction anthropologique devient possible. Montaigne ne pose un cadre de référence que pour le déborder et s'en échapper. La façon dont il dispose des schémas de pensée préétablis, topoi, mythes, sentences, fables antiques, comme celles de l'âge d'or, de l'Atlantide, de la

${ }^{271}$ Cf. Montaigne, Essais, III, 2, 805 : «Tant y a que je me contredits bien à l'adventure, mais la verité, comme disoit Demades, je ne la contredy point ». 
république idéale ou du philosophe nu, ressemble parfois à un jeu de massacre. Mais ce jeu n'a rien de gratuit. Que Montaigne aligne des lieux communs pour mieux en distinguer ensuite le cours de sa propre pensée ne doit pas faire conclure au pur plaisir de la destruction des fausses évidences - de leur déconstruction, dirait-on aujourd'hui. Dans ces déplacements et ce voyage continuel de la pensée, il y a bien la recherche d'une terre ferme du sens. En attendant, l'intelligence se meut sur un sol instable, terre meuble ou mer mobile, île inconstante emplie de voix et de démons.

Dans La Tempête, l'ingénieuse fiction exposée par Gonzalo ne suscite que l'irritation d'Alonso. Sa lassitude n'est nullement réconfortée par la déclamation de Gonzalo, bien au contraire. : «thou dost talk nothing to me». Comme le traduit Joseph Aynard: «tu ne dis que des riens ${ }^{272}$. Le rien, justement, ce pourrait être la définition lapidaire de la déclamation, un rien qui ouvre et déploie toutes les possibilités du sens.

\section{Les ém erveillements de Gonzalo}

Gonzalo ne cesse de s'émerveiller. À peine débarqué sur l'île, il se réjouit de ce sauvetage miraculeux, manifestant une joie telle qu'il indispose Alonso, tout à son deuil de Ferdinand, son fils perdu et peutêtre noyé. Le second émerveillement de Gonzalo touche à la fécondité de l'île: «Here is everything advantageous to life». «Ici on trouve toute chose avantageuse à la vie ${ }^{273}$. Écho probable de Montaigne : «Au demeurant, ils vivent en une contrée de païs tres-plaisante et bien temperée ; de façon qu'à ce que m'ont dit mes tesmoings, il est rare d'y voir un homme malade » ${ }^{274}$. Ricanements d'Antonio et de Sébastien, qui ne voient, au lieu du paradis évoqué par Gonzalo, que marécage et lande.

Troisième émerveillement de Gonzalo: leurs vêtements, en dépit de l'eau de la mer, sont intacts et plus frais qu'au mariage de Claribel à Tunis. Quatrième émerveillement: Tunis est bâtie à l'emplacement de l'ancienne Carthage, et le mariage de Claribel rappelle les amours d’Énée et de Didon, la «veuve Didon » chantée par

272 The Tempest, II.i. 159 , p. 63.

273 Ibid., II.i.46, p. 54-55.

274 Montaigne, Essais, I, 31, 207. 
Virgile et Ovide. Le rapprochement est pour le moins malencontreux, puisqu'il s'agit là d'amours glorieuses, sans doute, mais néanmoins tragiques. Il est peu probable qu'Alonso tire la moindre consolation de ce sujet de tragédie. Cet émerveillement, qui semble porter le comble à l'incongruité des propos de Gonzalo, donne peut-être la clé du personnage. Gonzalo croit au pouvoir des mots, à la différence de ses deux contradicteurs, des âmes noires qui n'ont que sarcasmes pour les légendes antiques: "His word is more than the miraculous harp » (II.i.78). D'où des moqueries en cascade, la métamorphose de l'île en pomme et les propos mirifiques de Gonzalo comparés à des pépins semés dans la mer et suscitant tout un archipel fabuleux.

Mais à partir de là, s'opère un retournement. Retrouvant le sens des réalités, Gonzalo s'élève contre Sébastien qui blâme Alonso d'avoir marié sa fille Claribel à Tunis en Afrique, au lieu de la réserver à un prince d'Europe, et s'est rendu par là coupable de ses malheurs. Il a de plein gré perdu sa fille, avant de perdre aussi son fils. Gonzalo s’indigne d'un reproche qui n'est pas sans fondement, mais se révèle totalement dépourvu de charité: "The truth you speak doth lack some gentleness » (II.i.126). Gonzalo, quant à lui, ne manque ni de compassion ni de «gentleness », de «gentillesse», comme aurait pu dire Montaigne, c'est-à-dire de noblesse. On commence à comprendre dès lors le fond de son caractère : l'amour des merveilles et le goût des fables antiques ne procèdent pas chez lui de l'admiration béate de l'érudit. Ces évocations légendaires, qui ne font rire que les méchants, donnent le change sur la cruauté de l'existence. Elles ont valeur compensatoire, même si elles manquent parfois leur but. C'est à ce moment précis du reste, que, hors de propos en apparence, Gonzalo pastiche Montaigne et récite la litanie négative des «Cannibales ». Pas plus que Montaigne, Gonzalo, en définitive, n'est dupe des prestiges de la fable, mais comme lui, il pense que la fable est nécessaire à notre infirmité, et pour ainsi dire consubstantielle à notre humanité. Humaniste, en ce sens profond, et sans illusion sur notre nature réelle, il pourrait reprendre à son compte la fameuse méditation de Prospero : «We are such stuff / As dreams are made on», "Nous sommes de l'étoffe dont sont faits les rêves » (IV.i.156-7).

Lucidité de Gonzalo : c'est lui qui, inspiré par Ariel penché sur son oreille, réveille le roi Alonso menacé et ses compagnons endormis : 
À la fin de la même scène, Gonzalo prononce des paroles à double sens, qui laissent entendre qu'il a bien perçu d'où vient le danger, non d'un lion ou d'une horde de lions, mais d'hommes pires que des lions :

Heavens keep him from these beasts !

Son personnage, dès lors, n'a plus rien de ridicule. Quand plus tard harassé et rhumatisant, il demande à se reposer, il rencontre la compassion d'Alonso, accablé lui aussi de fatigue et plus encore de douleur : «Old lord, I cannot blame thee » (III.iii.4).

Puis, quand résonne la musique céleste orchestrée par Prospero et qui suscite une nouvelle fois son admiration, il a cette autre phrase à double sens, que Prospero, invisible dans les airs, s'empresse de décoder. Les monstres de l'île sont «plus civils en leurs façons » que bien des hommes, en vérité que la plupart :

Their manners are more gentle, kind, than of

Our human generation you shall find

Many, nay, almost any.

(III.iii.32-4)

On voit donc combien Gonzalo est éloigné de l'optimisme béat qu'on serait tenté de lui prêter au premier abord. En même temps que le plus idéaliste, en apparence, des naufragés de l'île, c'est aussi, en vérité, le plus perspicace et le plus lucide - le plus cruellement lucide, devraiton dire. Sa fable politique peut paraître bien innocente. Elle ne l'est guère plus que celle de Montaigne, comme le montre la suite de l'intrigue. Car les émerveillements de Gonzalo n’interdisent pas de sa part la clairvoyance critique la plus crue.

Dès lors ses émerveillements ne font plus rire personne. Quand, face aux formes étranges suscitées par l'art magique de Prospero, Gonzalo se dit prêt à accorder tout crédit aux dires les plus extravagants des gens de mer, il mérite d'être cru lui-même sur parole. Rappelons à cet égard qu'en Angleterre plus longtemps peut-être qu'ailleurs en Europe, s'est maintenue la croyance dans les merveilles géographiques léguées par l'Antiquité et le Moyen Âge. À preuve la faveur dont jouit très tard le Voyage fabuleux de Jean de Mandeville, réédité jusque dans les Principall Navigations de Richard Hakluyt en $1589^{275}$, ou encore la Descriptio Regni Guianae de Sir Walter Raleigh,

275 Richard Hakluyt, The Principall Navigations, Voiages and Discoveries of the English Nation, made by Sea or over Land, to the most remote and farthest distant Quarters of the 
qui, à l'extrême fin du XVI ${ }^{\mathrm{e}}$ siècle, représente des Amazones archères et des Blemmies, de face et de dos, avec le visage au milieu de la poitrine ${ }^{276}$. Telle gravure illustrant cette étonnante «Description du royaume de la Guyane » est sans doute à l'origine directe du propos de Gonzalo concernant « such men / Whose heads stood in their breasts » (III.iii.46-7).

La naïveté apparente de Gonzalo n'est pas telle que l'on pourrait croire aujourd'hui. Sa faculté d'émerveillement, que ses nombreuses lectures n'ont pas entamée, bien au contraire, est celle de beaucoup de ses contemporains. Au cours de la même scène, du reste, Sébastien et Alonso l'ont précédé dans cette attitude d'admiration qui, devant un spectacle inouï et incompréhensible, renonce bien volontiers à tout esprit critique.

\section{La Servitude volontaire}

Il est un autre point où La Tempête rejoint la leçon des Cannibales de Montaigne. Le chapitre des Essais s'achevait par une entrevue entre trois Indiens débarqués du Brésil et le jeune roi Charles IX en visite à Rouen. Les premiers s'étonnaient d'un enfant roi commandant à de solides soldats armés de pied en cap. Par leur bouche, Montaigne faisait écho au paradoxe de la Servitude volontaire que La Boétie avait développé dans sa déclamation. Ce paradoxe peut se résumer ainsi : il est impossible à un homme seul, «nu et défait», d'asservir tout un peuple si ce peuple ne s'asservit pas d'abord lui-même. Or «c'est le peuple qui s'asservit, qui se coupe la gorge, qui, ayant le choix ou d'être serf ou d'être libre, quitte sa franchise et prend le joug». En toute rigueur La Boétie analysait les moyens - et notamment la pyramide des intérêts — dont s'aide le tyran pour demeurer au pouvoir et faire

earth, Londres, George Bishop and Ralph Newberie, 1589, p. 25-77: « The voyage of John Mandevill knight in latin, begun in the raigne of Edward the 2. Anno 1322, continued for the space of 33. yeeres, and ended in the raigne of Edward the 3. from England to Iudea and from thence to India, China, Tartaria, and as farre as 33 degrees to the south of the Aequinoctiall».

${ }^{276}$ Sir Walter Raleigh, Brevis et admiranda descriptio Regni Guianae, auri abundantissimi, in America, seu Novo Orbe, sub linea aequinoctilia siti : Quod nuper admodum, annis nimirum 1594. 1595 et 1596. Per generosum dominum, Dn Gualtherum Ralegh equitem Anglum detectum est : paulo post jusssu ejus duobus libellis comprehensa, Nuremberg, C. Lochner, pour Levinus Hulsius, 1599, p1. 15. La relation originale en anglais avait paru en 1596 à Londres chez R. Robinson, sous le titre: The discoverie of the large, rich, and bew tiful empyre of Guiana. 
que, de complicité en complicité, le corps social s'enchaîne lui-même. L'objet du Discours, c'est donc, fondamentalement, la politique en tant que telle 277

Voici que La Tempête ajoute au paradoxe de la Servitude volontaire une nouvelle illustration. Sans doute Caliban est-il d'abord esclave malgré lui, asservi par Prospero pour avoir tenté de violer sa fille Miranda. Mais croyant se libérer, il se rend esclave volontaire à Stephano. Il échange un maître pour un autre, plus brutal et qui est la caricature du premier, remplaçant le duc de Milan par son sommelier ivrogne. À l'instant même de son asservissement volontaire à ce personnage ridicule et méchant, qui veut se faire lui aussi roi de l'île, et alors même qu'il devient l'esclave d'un esclave, Caliban s'écrie: «Freedom, high-day! high-day, freedom ${ }^{278}$ !» Stephano a alors ce commentaire aussi exact que lapidaire: «O brave monster!» Indéniablement c'est un beau monstre que l'homme qui aliène sa liberté au plus vil de ses semblables.

Notons que le motif de la Servitude volontaire rencontre dans La Tempête sa variante amoureuse. Prisonnier de Prospero, le jeune Ferdinand se déclare l'esclave de Miranda, sa fille, dont il est tombé éperdument amoureux et qu'il demande en mariage :

Ay, with a heart as willing

As bondage e'er of freedom : here's my hand.

(III.i.89-90)

\section{Gonzalo's books}

De ses livres, Prospero tire ses pouvoirs surnaturels et donc une sorte de surhumanité. C'est par ses livres qu'il peut asservir les forces de la Nature et ravir à Jupiter même sa foudre. De manière significative, quand il s'apprête à quitter lî̀le de tous les sortilèges pour regagner l'Europe et Milan, il renonce à sa magie toute-puissante et jette son livre à la mer : «I'll drown my book » (v.i.57).

Les livres rapprochent Gonzalo et Prospero. On sait comment Gonzalo a aidé Prospero à emporter ses livres, lorsque ce dernier a été chassé de Milan, et comment d'une certaine manière il a sauvé ses livres - Prospero's books - les livres d'où Prospero tient son pouvoir

277 Voir Pierre Clastres et Claude Lefort, appendice à Étienne de La Boétie, Le Discours de la Servitude volontaire, Paris, Payot, 1976, p. 229-307.

278 The Tempest, II.ii. 160. 
sur la nature et sur les hommes ${ }^{279}$. Nul hasard si les adversaires de Gonzalo sont ceux de Prospero, si ces âmes noires d'Antonio et de Sébastien, et dans une moindre mesure d'Alonso, se moquent de Gonzalo et de ses références livresques. Ce sont des ennemis des livres, et donc de l'intelligence, et par conséquent de l'humanité en son plus haut sens.

Comme Prospero et comme Montaigne, Gonzalo entretient un commerce assidu avec les livres. Encore faut-il distinguer, car il y a livres et livres, d'un côté les grimoires qui permettent d'assujettir les éléments et les êtres, et de l'autre les livres d'histoires fabuleuses ou réelles dont la fréquentation engendre plutôt l'inadaptation au réel et au présent. Alors que Prospero tire de ses livres magiques sa toutepuissance, il semble que Gonzalo ne recueille de ses propres lectures que la faiblesse, voire l'impuissance inhérente au contemplatif - à l'intellectuel, dirions-nous aujourd'hui.

En fait, c'est toute l'humanitas de Gonzalo qui découle de ses livres. Son humanité plonge ses racines dans l'histoire et la philosophie naturelle des Anciens, Virgile et Pline, qu'il ne nomme pas, mais qu'il a lus et qu'il semble parfois réciter. C'est de ses lectures qu'il retire son admiration pour la veuve Didon, ou encore sa croyance dans les races monstrueuses décrites par Pline l'Ancien et les modernes cosmographes, Mandeville, Hakluyt et Raleigh.

Là où Prospero réalise l'idéal inaccessible de tout intellectuel, qui consiste à joindre l'action à la connaissance, Gonzalo ne peut que constater leur douloureux et risible divorce. C'est ce qui rend Gonzalo plus proche de Montaigne et de nous-mêmes. C'est ce qui tend à faire de lui la figure tour à tour ridicule et pathétique de l'humaniste confronté à un monde barbare. Que peuvent les pauvres humanités face à des bêtes («beasts») n'ayant d'hommes que la face, comme Antonio et Sébastien? La mémoire culturelle de l'Antiquité la plus haute et la plus prestigieuse apparaît bien dérisoire, quand il s'agit d'en tirer des leçons ou des remèdes pour le présent.

C'est un peu, si l'on en croit Claude Lévi-Strauss dans Histoire de lynx, la question que posent tout au long les Essais, et en particulier l'Apologie de Raimond Sebond:

279 The Tempest, I.ii, p. 22-23. 
La philosophie de Montaigne pose que toute certitude a la forme $a$ priori d'une contradiction, et qu'il n'y a rien à chercher par-dessous. La connaissance, l'action sont à jamais placées dans une situation fausse : prises entre deux systèmes de référence mutuellement exclusifs et qui s'imposent à elles, bien que la confiance même temporaire faite à l'un détruise la validité de l'autre. Il nous faut pourtant les apprivoiser pour qu'ils cohabitent en chacun de nous sans trop de drames. La vie est courte: c'est l'affaire d'un peu de patience. Le sage trouve son hygiène intellectuelle et morale dans la gestion lucide de cette schizophrénie ${ }^{280}$.

Comment résorber en effet le divorce entre la connaissance et l'action ? Là où Montaigne, en philosophe et en moraliste, établit un constat, Shakespeare, en homme de théâtre, suggère deux solutions. Ces deux moyens sont la magie, c'est-à-dire le théâtre, et l'ironie, qui suppose la distance, le jeu et le dédoublement, c'est-à-dire encore une fois le théâtre.

La magie de Prospero, personnage d'exception qui recourt à des moyens exceptionnels, c'est le pouvoir des livres, ou plutôt d'un livre, sur le monde. Reste que même là il convient de faire la part des choses. Car le monde sur lequel Prospero règne par son livre de magie se limite à une île, un espace clos et étroitement circonscrit, comme l'est la scène vivement éclairée d'un théâtre. «L'île est la métaphore de la scène et la scène la projection de l'univers », note Jean-Michel Racault, qui ajoute non moins justement: «La disposition architecturale du théâtre élisabéthain — un théâtre en rond entouré de galeries — ne peut que renforcer l'analogie ${ }^{281}$.

L'île de Prospero, c'est tout à la fois le modèle réduit et le simulacre du monde, un monde miniature sur lequel l'esprit, par le truchement des livres, peut avoir prise, au contraire du monde réel dont l'immensité et les troubles profondeurs le dépassent. C'est pourquoi Shakespeare a remplacé le «païs infini» des Cannibales de Montaigne par l'île de Prospero, objet topographique plus apte à se soumettre à l'emprise d'une intelligence supérieure et bienveillante.

280 Claude Lévi-Strauss, Histoire de lynx, Paris, Plon, 1991, p. 288.

${ }^{281}$ Voir sur ce point la lecture très convaincante de Jean-Michel Racault, «Insularité, théâtralité et pouvoir dans La Tempête de Shakespeare: pour une réévaluationde l'épilogue », in Le Cabinet de curiosités. Mélanges offerts à Claude Wanquet. Textes réunis par Colombe Couëlle, Paris, L'Harmattan, et Saint-Denis, Université de La Réunion, 2000 , p. 327-346. Citation, p. 345. Cf., dans un ordre d'idées voisin, Raymond Gardette, "Some vanity of mine art': l'espace magique dans La Tempête ", in Shakespeare, "La Tempête ". Études critiques; Actes du Colloque de Besançon, 2-3-4 décembre 1993, Besançon, Université de Franche-Comté, 1994, p. 21-37. 
Non que cette île soit une table rase : elle a ses zones d'ombre, ses forêts et ses marécages. C'est dans l'île que Sycorax la sorcière a accouché du noir Caliban, avant d'y être enterrée avec tous ses charmes puissants. Y débarquent ensuite deux fières fripouilles et un couple d'ivrognes, Antonio et Sébastien, Stéphano et Trinculo. Le mal, indiscutablement, est dans l'île. Il l'habite. Mais il n'en a pas encore pris complètement possession. Et c'est le pardon qui vaincra.

La magie, le théâtre, prendront fin, quand les personnages quitteront l'île des songes pour regagner la terre ferme du réel. Il faut alors composer. Par le pardon et par l'ironie. Faire comme si le songe, cette étoffe dont nous sommes faits, n'était pas incompatible avec le réel et pouvait se transvaser en lui. À la fin de la pièce, le pardon de Prospero à ses adversaires vaincus, dont la méchanceté a été amplement démontrée et confirmée tout au long de l'action, est une manière, nullement dupe, de réconcilier l'idéal avec les réalités fort inhumaines qui sommeillent en chacun de nous. Gonzalo continuera de s'émerveiller, tout en sachant bien que son admiration peut être à tout instant prise en défaut, démentie par l'abîme vertigineux d'une réalité ingrate.

De même Montaigne pour qui «les miracles sont selon l'ignorance en quoi nous sommes de la nature, non selon l'être de la nature » $(\mathrm{I}, 26)^{282}$. À la suite de saint Augustin, Montaigne redonne au miracle son sens premier, son sens profond, lié à l'étonnement devant la variété du monde et du moi: «Je n'ay veu monstre et miracle au monde plus expres que moy-mesme. On s'apprivoise à toute estrangeté par l'usage et le temps; mais plus je me hante et me connois, plus ma difformité m'estonne, moins je m'entens en moy » ${ }^{283}$. Sur les ruines de l'orgueil dogmatique, c'est l'homme concret qui apparaît, dans toute sa complexité, comme source de merveille.

Montaigne nous invite donc, selon Jean Céard, à «nous désaccoutumer de nous-même et à réveiller en nous l'aptitude à l'étonnement ${ }^{284}$. Iris [la messagère des dieux] n'est-elle pas « fille de Thaumantis », le centaure Thaumas, dont le nom évoque le verbe grec

\footnotetext{
282 Voir Jean Céard, La Nature et les prodiges. L'Insolite au XVI siècle, en France, Genève, Droz, 1977, VI ${ }^{\mathrm{e}}$ Partie, chap. XVII, p. 409 sqq.

283 Montaigne, Essais, III, 11, 1029.

284 J. Céard, op. cit., p. 426.
} 
«admirer»? Montaigne dit encore : «L'admiration est fondement de toute philosophie, l'inquisition le progrez, l'ignorance le bout $»^{285}$.

C'est ainsi que l'on pourrait comprendre la conversion d'Antonio et de Sébastien au miracle, à l'émerveillement. Longtemps moqueurs à l'égard de Gonzalo, ils finissent par partager son admiration. Quand, à l'acte III, Prospero, avec le concours d'Ariel, produit devant eux un théâtre d'esprits qui ressemble à un théâtre de marionnettes, ils tombent à leur tour béats d'admiration. Sébastien s'écrie : «Maintenant je croirai qu'il y a des licornes». Antonio lui fait écho: «Je veux jurer que c'est vrai. Les voyageurs ne mentent jamais, quoique les sots, restés à la maison, les condamnent ${ }^{286}$. À compter de cet instant, ils sont sauvés - et pour cette raison pardonnés. D’une certaine manière, Prospero les a conquis à la cause de Gonzalo, qui est aussi et d'abord la sienne. La cause des livres qui, par leurs fables, ouvrent au monde inépuisable de l'imagination et rendent la vie tout à la fois intelligible et supportable, même s'ils n'y préparent pas toujours de la manière la plus directe et la plus aisée.

La cause d'un livre. Le livre de Gonzalo, c'est Montaigne. Les Essais de Montaigne : Gonzalo's books.

Frank LESTRINGANT Université de Paris IV - Sorbonne

285 Montaigne, Essais, III, 11, 1030

286 Shakespeare, The Tempest, III.iii.21-2, 26-7. 